\title{
Pricing Options and Convertible Bonds Based on an Actuarial Approach
}

\author{
Jian Liu, ${ }^{1,2}$ Lizhao Yan, ${ }^{3}$ and Chaoqun $\mathrm{Ma}^{1}$ \\ ${ }^{1}$ Business School, Hunan University, Changsha 410082, China \\ ${ }^{2}$ School of Economics and Management, Changsha University of Science and Technology, Changsha 410004, China \\ ${ }^{3}$ Hunan Normal University Press, Changsha 410081, China
}

Correspondence should be addressed to Chaoqun Ma; cqma@hnu.cn

Received 2 September 2013; Accepted 19 October 2013

Academic Editor: Fenghua Wen

Copyright (c) 2013 Jian Liu et al. This is an open access article distributed under the Creative Commons Attribution License, which permits unrestricted use, distribution, and reproduction in any medium, provided the original work is properly cited.

\begin{abstract}
This paper discusses the pricing problem of European options and convertible bonds using an actuarial approach. We get the pricing formula of European options, extend the pricing results to the case with continuous dividend, and then derive the call-put parity relation. Furthermore, we get the general expression of convertible bond price. Finally, we conduct a comparative analysis of numerical simulation and make an empirical analysis between the B-S model and the actuarial model using the actual data in the Chinese stock market. The empirical results show that the efficiency of the actuarial model is superior to the B-S model.
\end{abstract}

\section{Introduction}

The contingent claim pricing has always been one of the core subjects in the field of financial engineering research. To price contingent claims correctly and scientifically is the basis of financial risk management as well as an indispensable component of modern finance. Black and Scholes [1] derived the well-known Black-Scholes (B-S) formula using arbitrage reasoning and stochastic analysis in their classic paper on option pricing and thus established the option pricing theory. The B-S pricing model has had a huge influence on the financial theory. However, it was based on multiple assumptions, such as a normal distribution for the underlying asset price process, no divided paying for the underlying asset, and the constant risk-free interest rate, which apparently do not fit in with the ever-changing financial market. Later, a number of researchers improved and popularized the B-S model. Merton [2] validated the B-S formula using the arbitrage theory and derived the analytic solution to the continuous time model for optimal consumption and investment decision using dynamic programming methods. Cox et al. [3] proposed the binominal option pricing model. Duffie [4] made further inference for the B-S formula with the conventional option pricing approach. Liu and Zhao [5] develop an efficient lattice approach for option pricing. Ammann et al. [6] propose and empirically investigate a pricing model for convertible bonds based on the enhanced Monte Carlo simulation. Xu [7] uses a lattice approach to pricing the convertible bond asset swap.

Most of the existent research on option pricing was based on martingale measure or numerical simulation theory in the framework of Black-Scholes option pricing approach and is only applicable in a complete financial market, where all contingent claims are capable of being replicated accurately with the asset portfolios available on the existent financial markets; that is to say, the market is in an equilibrium, arbitrage-free, and one and only equivalent martingale measure exists. But the complete market assumption may not be perfectly relevant to the actual investment environment. If the financial market is incomplete, assets cannot be accurately replicated or hedged like in a complete market. A common alternative practice is to seek a family of final wealth derived from self-financing strategies to approach the value of the asset, which naturally entails errors. For example, Follmer and Sondermann [8] proposed the "mean-variance" criterion to measure the error, but the solution process is rather complicated. 
Bladt and Rydberg [9] first proposed an actuarial approach to pricing options, which transforms the option pricing into a problem equivalent to determining the fair insurance premium. As no economic assumptions are involved, this approach is valid for incomplete markets as well as for complete markets, and demonstrated that the price derived whereby is consistent with that from the B-S model in the continuous time case. Yan and Liu [10] used the actuarial approach to derive the European option pricing formula where the stock price is assumed to follow the OrnsteinUhlenbeck (O-U) process, and Zhao and He [11] studied the option pricing model where the stock price is assumed to follow a fractional $\mathrm{O}-\mathrm{U}$ process in a risk neutral market. The assumption of the stock price following the $\mathrm{O}-\mathrm{U}$ process avoids the limitation that the stock price tends to change in one direction under the lognormal distribution assumption and weakens the tendency of the stock price rise. But the assumption for the above model that the interest rate is a deterministic function of time cannot satisfy the requirement of the actual conditions of the market. A number of empirical lines of evidence show that, in real financial markets, the interest rate has the property of mean-reversion, the volatility of the long-term interest rate is less than that of the short-term interest rate, and the volatility is greater when the interest rate is relatively higher $[12,13]$. Liu et al. [14] studied the reload stock option pricing under the assumption of the interest rate following the Hull-White model with martingale pricing method. The stochastic interest rate model assumes that the interest rate converges with the time at a certain mean reversion level.

The content of this paper is arranged as follows. In Section 2, we make some basic assumption for the financial market where the stock prices are driven by $\mathrm{O}-\mathrm{U}$ process and the interest rates are driven by Hull-White model. In Section 3, we consider the models in the continuous time and apply the actuarial approach to price the European option and the convertible bond. To show the role that the actuarial approach and the stochastic interest rates play, we conduct a comparative analysis of numerical simulation in Section 4. As in Section 5, we make an empirical analysis between the B-S model and the actuarial model, using the actual data in the Chinese stock market. Section 6 concludes the paper.

\section{Basic Assumption}

Suppose that the financial market is frictionless and continuous, and there are two assets. The risky asset is the stock and the risk-free asset is the bond. A complete probability space $\left(\Omega, F,\left\{F_{t}\right\}_{t \geq 0}, P\right)$ describes the financial market where the filtration satisfies the usual conditions.

Assume that the stock price $S(t)$ follows the OrnsteinUhlenback process as follows:

$$
\begin{aligned}
d S(t)= & (\mu(t)-\alpha \ln S(t)) S(t) d t \\
& +\sigma_{s}(t) S(t) d B(t), \quad S(0)=S,
\end{aligned}
$$

where $S>0$ and $\sigma_{S}(t)$ is the volatility of the stock price. $\alpha$ is a constant and $\mu(t)$ and $\sigma_{S}(t)$ are some determinate functions of $t$.
The short-term interest rate $r(t)$ follows the Hull-White model as follows:

$$
d r(t)=(a(t)-b(t) r(t)) d t+\sigma_{r}(t) d W(t), \quad r(0)=r,
$$

where the parameters $a(t), b(t)$, and $\sigma_{r}(t)$ are some determinate functions of time $t$. The Hull-White model is the mean-reversion model, where the parameter $a(t)$ is the longterm average level, and $b(t)$ is the average reversion rate of interest rates. When the parameters $a(t)$ and $b(t)$ are constant, the Hull-White model (2) becomes the Vasicěk model. The stochastic processes $\{B(t): t \geq 0\}$ and $\{W(t): t \geq 0\}$ are two standard Brownian Motions in the defined probability space $\left(\Omega, F,\left\{F_{t}\right\}_{t \geq 0}, P\right)$, and their correlation coefficient is supposed to be $\rho$.

Now, we give two definitions of the actuarial approach.

Definition 1 (see [9]). The expected yield rate of the stock price process $\{S(t), 0 \leq t \leq T\}$ in the time interval $[0, T]$ is defined by $\int_{0}^{T} \beta(t) d t$ which satisfies the following equation:

$$
e^{\int_{0}^{T} \beta(t) d t}=\frac{E[S(T)]}{S} .
$$

Definition 2 (see [9]). Suppose the expiration date of the European option is $T$ and the strike price is $K$. In the actuarial approach, the stock price is discounted by the expected yield rate defined in (3), and the strike price is discounted by the riskless interest rate on maturity. The European option value is defined as the expectation of the difference which is between the two discount values on the actual probability measure as the option is exercised. The sufficient and necessary condition for exercising the European call option on $T$ is

$$
\exp \left\{-\int_{0}^{T} \beta(t) d t\right\} S(T)>\exp \left\{-\int_{0}^{T} r(t) d t\right\} K,
$$

and the similar condition to the European put option is

$$
\exp \left\{-\int_{0}^{T} \beta(t) d t\right\} S(T)<\exp \left\{-\int_{0}^{T} r(t) d t\right\} K
$$

Let $C(K, T)$ denote the call value and $P(K, T)$ denote the put value at the time 0 . Then, in the actuarial approach, the two options values are defined as follows, respectively:

$$
\begin{aligned}
& C(K, T)=E\left[\left(\exp \left\{-\int_{0}^{T} \beta(t) d t\right\} S(T)\right.\right. \\
& \left.-\exp \left\{-\int_{0}^{T} r(t) d t\right\} K\right) \\
& \left.\times 1_{\left\{\exp \left\{-\int_{0}^{T} \beta(t) d t\right\} S(T)>\exp \left\{-\int_{0}^{T} r(t) d t\right\} K\right\}}\right], \\
& P(K, T)=E\left[\left(\exp \left\{-\int_{0}^{T} r(t) d t\right\} K\right.\right.
\end{aligned}
$$




$$
\begin{gathered}
\left.-\exp \left\{-\int_{0}^{T} \beta(t) d t\right\} S(T)\right) \\
\left.\times 1_{\left\{\exp \left\{-\int_{0}^{T} \beta(t) d t\right\} S(T)<\exp \left\{-\int_{0}^{T} r(t) d t\right\} K\right\}}\right] .
\end{gathered}
$$

\section{Pricing Formula}

In this section, we firstly consider the pricing problem of the European options in the financial market models described above. Then we extend the pricing result to the options whose underlying asset has continuous dividend and derive the callput parity relation using the actuarial approach. Furthermore, we get the general expression of convertible bond price. At first, we offer two important lemmas.

Lemma 3 (see [15]). If the random variables $W_{1}$ and $W_{2}$ are both standard normally distributed with mean 0 and variance 1 notated as $N(0,1)$, and their covariance is $\operatorname{Cov}\left(W_{1}, W_{2}\right)=\rho$, then for any real numbers $a, b, c, d$, and $k$ one has the following equation:

$$
\begin{aligned}
& E\left[e^{c W_{1}+d W_{2}} 1_{\left\{a W_{1}+b W_{2} \geq k\right\}}\right] \\
& =e^{(1 / 2)\left(c^{2}+d^{2}+2 \rho c d\right)} N\left(\frac{a c+b d+\rho(a d+b c)-k}{\sqrt{a^{2}+b^{2}+2 \rho a b}}\right) .
\end{aligned}
$$

Lemma 4. If the stock price $S(t)$ is driven by the $O-U$ process (1), then one gets the following equations:

$$
\begin{array}{r}
S(t)=S^{e^{-\alpha t}} \exp \left\{e^{-\alpha t} \int_{0}^{t}\left(\mu(t)-\frac{1}{2} \sigma_{s}^{2}(u)\right) e^{\alpha u} d u\right. \\
\left.+e^{-\alpha t} \int_{0}^{t} \sigma_{s}(u) e^{\alpha u} d B(u)\right\}, \\
E[s(t)]=S^{e^{-\alpha t}} \exp \left\{e^{-\alpha t} \int_{0}^{t}\left(\mu(t)-\frac{1}{2} \sigma_{s}^{2}(u)\right) e^{\alpha u} d u\right. \\
\left.+\frac{1}{2} e^{-2 \alpha t} \int_{0}^{t} \sigma_{s}^{2}(u) e^{2 \alpha u} d B(u)\right\} .
\end{array}
$$

Proof. With the Itŏ formula, we get

$$
\begin{aligned}
d \ln S(t)= & \frac{1}{S(t)} d S(t)-\frac{1}{2 S^{2}(t)} d S(t) \\
= & \left(\mu(t)-\alpha \ln S(t)-\frac{1}{2} \sigma_{s}^{2}(t)\right) d t \\
& +\sigma_{s}(t) d B(t), \\
d\left(\ln S(t) e^{\alpha t}\right)= & \left(\mu(t)-\frac{1}{2} \sigma_{s}^{2}(t)\right) e^{\alpha t} d t+\sigma_{s}(t) e^{\alpha t} d B(t),
\end{aligned}
$$

thus, we get

$$
\begin{aligned}
\ln S(t) e^{\alpha t}= & \ln S+\int_{0}^{t}\left(\mu(u)-\frac{1}{2} \sigma_{s}^{2}(u)\right) e^{\alpha u} d u \\
& +\int_{0}^{t} \sigma_{s}(u) e^{\alpha u} d B(u) .
\end{aligned}
$$

Then

$$
\begin{aligned}
\ln S(t)= & e^{-\alpha t} \ln S+e^{-\alpha t} \int_{0}^{t}\left(\mu(u)-\frac{1}{2} \sigma_{s}^{2}(u)\right) e^{\alpha u} d u \\
& +\int_{0}^{t} \sigma_{s}(u) e^{\alpha u} d B(u),
\end{aligned}
$$

so we have

$$
\begin{gathered}
S(t)=S^{e^{-\alpha t}} \exp \left\{e^{-\alpha t} \int_{0}^{t}\left(\mu(u)-\frac{1}{2} \sigma_{s}^{2}(u)\right) e^{\alpha u} d u\right. \\
\left.+e^{-\alpha t} \int_{0}^{t} \sigma_{s}(u) e^{\alpha u} d B(u)\right\} .
\end{gathered}
$$

Taking expectations on both sides of the above equation, then we get

$$
\begin{array}{r}
E[S(t)]=S^{e^{-\alpha t}} \exp \left\{e^{-\alpha t} \int_{0}^{t}\left(\mu(u)-\frac{1}{2} \sigma_{s}^{2}(u)\right) e^{\alpha u} d u\right. \\
\left.+\frac{1}{2} e^{-2 \alpha t} \int_{0}^{t} \sigma_{s}^{2}(u) e^{2 \alpha u} d B(u)\right\} .
\end{array}
$$

3.1. European Options Pricing. We consider the European options, call and put, whose underlying assets are stocks. Suppose that the exercise date of the options is $T$ and the strike price is $K$.

Theorem 5. Assume that the short-term interest rate is described by the Hull-White model and the stock process $\{S(t)$, $t \geq 0\}$ follows the $O-U$ process, then, at the time 0 , the pricing formulas of the call and put are as follows, respectively:

$$
\begin{aligned}
& C(K, T)=S N\left(d_{1}\right)-K \exp \left\{\frac{1}{2} \sigma_{X}^{2}-G(0, T)\right\} N\left(d_{2}\right), \\
& P(K, T)=K \exp \left\{\frac{1}{2} \sigma_{X}^{2}-G(0, T)\right\} N\left(-d_{2}\right)-S N\left(-d_{1}\right),
\end{aligned}
$$

where

$$
\begin{aligned}
n(t) & =\int_{0}^{t} b(t) d t, \quad m(t, T)=\int_{0}^{T} e^{n(t)-n(s)} d s \\
G(0, T) & =r m(0, T)+\int_{0}^{T} a(t) m(t, T) d t \\
X & =\int_{0}^{T} \sigma_{r}(t) m(t, T) d W(t) \\
Y & =e^{-\alpha T} \int_{0}^{T} \sigma_{s}(t) e^{\alpha t} d B(t)
\end{aligned}
$$




$$
\begin{aligned}
& \sigma_{X}^{2}=\int_{0}^{T} \sigma_{r}^{2}(t) m^{2}(t, T) d t \\
& \sigma_{Y}^{2}=e^{-2 \alpha T} \int_{0}^{T} \sigma_{s}^{2}(t) e^{2 \alpha t} d t \\
& d_{1}=\frac{\ln (S / K)+G(0, T)+(1 / 2) \sigma_{Y}^{2}+\rho \sigma_{X} \sigma_{Y}}{\sqrt{\sigma_{X}^{2}+\sigma_{Y}^{2}+2 \rho \sigma_{X} \sigma_{Y}}}, \\
& d_{2}=\frac{\ln (S / K)+G(0, T)-\sigma_{X}^{2}-(1 / 2) \sigma_{Y}^{2}+\rho \sigma_{X} \sigma_{Y}}{\sqrt{\sigma_{X}^{2}+\sigma_{Y}^{2}+2 \rho \sigma_{X} \sigma_{Y}}} .
\end{aligned}
$$

Proof. By Definition 2, we get

$$
\begin{aligned}
C(K, T)=E\left[S(T) \exp \left\{-\int_{0}^{T} \beta(t) d t\right\}\right. \\
\left.\cdot 1_{\left.\left\{S(T) \exp \left\{-\int_{0}^{T} \beta(t) d t\right\}>K \exp \left\{-\int_{0}^{T} r(t) d t\right\}\right\}\right]}\right] \\
-E\left[K \exp \left\{-\int_{0}^{T} r(t) d t\right\}\right. \\
\left.\cdot 1_{\left\{S(T) \exp \left\{-\int_{0}^{T} \beta(t) d t\right\}>K \exp \left\{-\int_{0}^{T} r(t) d t\right\}\right\}}\right] \\
=I_{1}-I_{2} .
\end{aligned}
$$

In the actuarial approach, the expected yield rate of the stock price satisfies (3), and, by Lemma 4, we have

$$
\begin{aligned}
\int_{0}^{T} \beta(t) d t= & \ln \frac{E[S(T)]}{S}=\left(e^{-\alpha T}-1\right) \ln S \\
& +e^{-\alpha T} \int_{0}^{T}\left(\mu(t)-\frac{1}{2} \sigma_{s}^{2}(t)\right) e^{\alpha t} d t \\
& +\frac{1}{2} e^{-2 \alpha T} \int_{0}^{T} \sigma_{s}^{2}(t) e^{2 \alpha t} d t .
\end{aligned}
$$

So,

$$
\begin{aligned}
e^{-\int_{0}^{T} \beta(t) d t}=\exp \{ & \left(1-e^{-\alpha T}\right) \ln S-e^{-\alpha T} \\
\times & \int_{0}^{T}\left(\mu(t)-\frac{1}{2} \sigma_{s}^{2}(t)\right) e^{\alpha t} d t \\
& \left.-\frac{1}{2} e^{-2 \alpha T} \int_{0}^{T} \sigma_{s}(t) e^{2 \alpha t} d t\right\}, \\
e^{-\int_{0}^{T} \beta(t) d t} S(T)=\exp \{ & \ln S-\frac{1}{2} e^{-2 \alpha T} \int_{0}^{T} \sigma_{s}^{2}(t) e^{2 \alpha t} d t \\
& \left.+e^{-\alpha T} \int_{0}^{T} \sigma_{s}(t) e^{\alpha t} d B(t)\right\} .
\end{aligned}
$$

By Itŏ formula and [14], the interest rate model (2) satisfies

$$
\int_{0}^{T} r(t) d t=G(0, T)+\int_{0}^{T} \sigma_{r}(t) m(t, T) d W(t) .
$$

The exercising condition $S(T) \exp \left\{-\int_{0}^{T} \beta(t) d t\right\}>K$ $\exp \left\{-\int_{0}^{T} r(t) d t\right\}$ is equal to

$$
\begin{aligned}
\exp \left\{\ln S-\frac{1}{2} e^{-2 \alpha T} \int_{0}^{T} \sigma_{s}^{2}(t) e^{2 \alpha t} d t\right. \\
\left.+e^{-\alpha T} \int_{0}^{T} \sigma_{s}(t) e^{\alpha t} d B(t)\right\}>K \\
\times \exp \left\{G(0, T)+\int_{0}^{T} \sigma_{r}(t) m(t, T) d W(t)\right\}, \\
\Longleftrightarrow \int_{0}^{T} \sigma_{r}(t) m(t, T) d W(t) \\
\quad+e^{-\alpha T} \int_{0}^{T} \sigma_{s}(t) e^{\alpha t} d B(t) \\
>\ln \frac{K}{S}-G(0, T)+\frac{1}{2} e^{-2 \alpha T} \\
\quad \times \int_{0}^{T} \sigma_{s}^{2}(t) e^{2 \alpha t} d t, \\
\Longleftrightarrow X+Y>\ln \frac{K}{S}-G(0, T)+\frac{1}{2} \sigma_{Y}^{2} .
\end{aligned}
$$

Because $\{B(t): t \geq 0\}$ and $\{W(t): t \geq 0\}$ are two standard Brownian Motions in the probability space $(\Omega$, $\left.\mathscr{F},\left\{\mathscr{F}_{t}\right\}_{t \geq 0}, P\right)$, so the defined normal random variables $X$ and $Y$ are independent from $\mathscr{F}_{t}$ and satisfy the following equations:

$$
\begin{gathered}
E^{Q}[X]=E^{Q}[Y]=0, \quad \sigma_{X}^{2}=\int_{0}^{T} \sigma_{r}^{2}(t) m^{2}(t, T) d t \\
\sigma_{Y}^{2}=e^{-2 \alpha T} \int_{0}^{T} \sigma_{s}^{2}(t) e^{2 \alpha t} d t .
\end{gathered}
$$

So, by Lemma 3, we get

$$
\begin{aligned}
I_{1}= & E\left[S \exp \left\{-\frac{1}{2} e^{-2 \alpha T} \int_{0}^{T} \sigma_{s}^{2}(t) e^{2 \alpha t} d t+Y\right\}\right. \\
& \left.\cdot 1_{\left\{X+Y>\ln (K / S)-G(0, T)+(1 / 2) \sigma_{Y}^{2}\right\}}\right] \\
= & S \exp \left\{-\frac{1}{2} e^{-2 \alpha T} \int_{0}^{T} \sigma_{s}^{2}(t) e^{2 \alpha t} d t\right\} \\
& E \cdot\left[e^{Y} \cdot 1_{\left\{X+Y>\ln (K / S)-G(0, T)+(1 / 2) \sigma_{Y}^{2}\right\}}\right] \\
= & S N\left(d_{1}\right), \\
I_{2}= & K e^{-G(0, T)} \\
& \cdot E\left[e^{-X} \cdot 1_{\left\{X+Y>\ln (K / S)-G(0, T)+(1 / 2) \sigma_{Y}^{2}\right\}}\right] \\
= & K \exp \left\{\frac{1}{2} \sigma_{X}^{2}-G(0, T)\right\} N\left(d_{2}\right) .
\end{aligned}
$$


Thus, the value of the call option is

$$
C(K, T)=S N\left(d_{1}\right)-K \exp \left\{\frac{1}{2} \sigma_{X}^{2}-G(0, T)\right\} N\left(d_{2}\right) .
$$

Similarly, we get the value of put option as follows:

$$
P(K, T)=K \exp \left\{\frac{1}{2} \sigma_{X}^{2}-G(0, T)\right\} N\left(-d_{2}\right)-S N\left(-d_{1}\right) .
$$

Furthermore, we deduce the following pricing inferences.

Inference 1. Suppose that the stock process $\{S(t), t \geq 0\}$ follows the $\mathrm{O}-\mathrm{U}$ process (1), and the interest rate is described by the Hull-White model (2); then the call-put parity of the European options in the actuarial approach is

$$
C(K, T)+K \exp \left\{\frac{1}{2} \sigma_{X}^{2}-G(0, T)\right\}=P(K, T)+S .
$$

Inference 2. Under the market models (1) and (2), the underlying stock has continuous dividend yield marked by $q(t)$. Then, respectively, the pricing formulas of the call and put option in the actuarial approach at time 0 are

$$
\begin{aligned}
C^{\prime}(K, T)= & S \exp \left\{-\int_{0}^{T} q(t) d t\right\} N\left(d_{1}^{\prime}\right) \\
& -K \exp \left\{\frac{1}{2} \sigma_{X}^{2}-G(0, T)\right\} N\left(d_{2}^{\prime}\right), \\
P^{\prime}(K, T)= & K \exp \left\{\frac{1}{2} \sigma_{X}^{2}-G(0, T)\right\} N\left(-d_{2}^{\prime}\right) \\
& -S \exp \left\{-\int_{0}^{T} q(t) d t\right\} N\left(-d_{1}^{\prime}\right),
\end{aligned}
$$

where

$$
\begin{aligned}
& d_{1}^{\prime}= \frac{\ln (S / K)+G(0, T)-\int_{0}^{T} q(t) d t+(1 / 2) \sigma_{Y}^{2}+\rho \sigma_{X} \sigma_{Y}}{\sqrt{\sigma_{X}^{2}+\sigma_{Y}^{2}+2 \rho \sigma_{X} \sigma_{Y}}} \\
& d_{2}^{\prime}=\left(\ln \left(\frac{S}{K}\right)+G(0, T)-\int_{0}^{T} q(t) d t\right. \\
&\left.\quad-\sigma_{X}^{2}-\frac{1}{2} \sigma_{Y}^{2}+\rho \sigma_{X} \sigma_{Y}\right) \\
& \quad \times\left(\sqrt{\sigma_{X}^{2}+\sigma_{Y}^{2}+2 \rho \sigma_{X} \sigma_{Y}}\right)^{-1} .
\end{aligned}
$$

3.2. Convertible Bond Pricing. We consider the pricing problem of convertible bond with the call provision. It is based on the actuarial approach where the stock price is driven by $\mathrm{O}$ U process and the interest rate follows Hull-White model. For the callable convertible bonds, based on the classical theory of optimal investment strategy, Brennan and Schwartz [16] and Ingersoll [17], the investors will not exercise the right of conversion while the issuers will immediately exercise the right of redemption when the stock prices reach the callable price for the first time [18]. Let $T$ be the maturity date of the convertible bond, and let $\tau^{*}$ be the first time when the stock price goes up to the callable trigger price; then the optimal exercise time $\tau=\tau^{*}$; otherwise, $\tau=T$.

Theorem 6. Suppose that the face value of the convertible bond is $B_{F}$, the conversion price is $K_{1}$, and the callable trigger price is $K_{2}$; then the general expression of convertible bond price $H$ is given by

$$
\begin{aligned}
H= & E\left[e^{-\int_{0}^{\tau^{*}} \beta(t) d t} \times\left(K_{2} \frac{B_{F}}{K_{1}}+c\left(\tau^{*}\right)\right) \times 1_{\left\{\tau^{*} \leq T\right\}}\right] \\
& +\left[\frac{B_{F}}{K_{1}} S N\left(d_{3}\right)+c(T) \exp \left\{\frac{1}{2} \sigma_{X}^{2}-G(0, T)\right\} N\left(d_{4}\right)\right] \\
& \times P\left(\tau^{*}>T\right) \\
& +\left[\left(B_{F}+c(T)\right) \cdot \exp \left\{\frac{1}{2} \sigma_{X}^{2}-G(0, T)\right\} N\left(-d_{4}\right)\right] \\
& \times P\left(\tau^{*}>T\right),
\end{aligned}
$$

where

$$
\begin{gathered}
d_{3}=\frac{\ln \left(S / K_{1}\right)+G(0, T)+(1 / 2) \sigma_{Y}^{2}+\rho \sigma_{X} \sigma_{Y}}{\sqrt{\sigma_{X}^{2}+\sigma_{Y}^{2}+2 \rho \sigma_{X} \sigma_{Y}}}, \\
d_{4}=\frac{\ln \left(S / K_{1}\right)+G(0, T)-\sigma_{X}^{2}-(1 / 2) \sigma_{Y}^{2}+\rho \sigma_{X} \sigma_{Y}}{\sqrt{\sigma_{X}^{2}+\sigma_{Y}^{2}+2 \rho \sigma_{X} \sigma_{Y}}} .
\end{gathered}
$$

Proof. As $\tau^{*}$ is the first time when the stock price goes up to the callable trigger price, so $\tau^{*}=\inf \left\{t, S_{t} \geq K_{2}\right\}$. Under the optimal investment strategy, the optimal execution time $\tau$ is

$$
\tau= \begin{cases}\tau^{*}, & \text { trigger before the expiry, } \\ T, & \text { no trigger before the expiry. }\end{cases}
$$

Let $c(t)$ express the time $t$ present value of the interest income before $t$; then the theoretical value of the convertible bond at the time $\tau$ defined by $g(r, S, \tau)$ is given by

$$
g(r, S, \tau)= \begin{cases}\frac{B_{F}}{K_{1}} K_{2}+c\left(\tau^{*}\right), & \tau^{*} \leq T, \\ \frac{B_{F}}{K_{1}} S_{T}+c(T), & \tau^{*}>T, S_{T}>K_{1}, \\ B_{F}+c(T), & \tau^{*}>T, S_{T} \leq K_{1} .\end{cases}
$$


Then the actuarial price of convertible bond $H(r, S, t)$ can be expressed as follows:

$$
\begin{array}{r}
H(r, S, t)=E\left[e^{-\int_{0}^{\tau^{*}} \beta(t) d t} \times\left(K_{2} \frac{B_{F}}{K_{1}}+c\left(\tau^{*}\right)\right) \times 1_{\left\{\tau^{*} \leq T\right\}}\right] \\
+E\left[e^{-\int_{t}^{T} \beta(t) d t} \times\left(S_{T} \frac{B_{F}}{K_{1}}+c(T)\right)\right. \\
\left.\quad \times 1_{\left\{\tau^{*}>T, \exp \left\{-\int_{0}^{T} \beta(t) d t\right\} S(T)>\exp \left\{-\int_{0}^{T} r(t) d t\right\} K_{1}\right\}}\right] \\
+E\left[e^{-\int_{t}^{T} \beta(t) d t} \times\left(B_{F}+c(T)\right)\right. \\
\left.\quad \times 1_{\left\{\tau^{*}>T, \exp \left\{-\int_{0}^{T} \beta(t) d t\right\} S(T) \leq \exp \left\{-\int_{0}^{T} r(t) d t\right\} K_{1}\right\}}\right],
\end{array}
$$

where $e^{\int_{0}^{\tau^{*}} \beta(t) d t}=E\left[S\left(\tau^{*}\right)\right] / S$.

From Theorem 5, we get

$$
\begin{aligned}
& e^{-\int_{0}^{\tau^{*}} \beta(t) d t} \\
& =\exp \left\{\left(1-e^{-\alpha \tau^{*}}\right) \ln S-e^{-\alpha \tau^{*}}\right. \\
& \times \int_{0}^{\tau^{*}}\left(\mu(t)-\frac{1}{2} \sigma_{s}^{2}(t)\right) e^{\alpha t} d t \\
& \left.-\frac{1}{2} e^{-2 \alpha \tau^{*}} \int_{0}^{\tau^{*}} \sigma_{s}(t) e^{2 \alpha t} d t\right\}, \\
& H_{1} \triangleq E\left[e^{-\int_{0}^{\tau^{*}} \beta(t) d t} \times\left(K_{2} \frac{B_{F}}{K_{1}}+c\left(\tau^{*}\right)\right) \times 1_{\left\{\tau^{*} \leq T\right\}}\right] \text {, } \\
& H_{2} \triangleq E\left[e^{-\int_{0}^{T} \beta(t) d t} \times\left(S_{T} \frac{B_{F}}{K_{1}}+c(T)\right)\right. \\
& \left.\times 1_{\left\{\tau^{*}>T, \exp \left\{-\int_{0}^{T} \beta(t) d t\right\} S(T)>\exp \left\{-\int_{0}^{T} r(t) d t\right\} K_{1}\right\}}\right] \\
& =\left[\frac{B_{F}}{K_{1}} S N\left(d_{3}\right)+c(T) \exp \left\{\frac{1}{2} \sigma_{X}^{2}-G(0, T)\right\} N\left(d_{4}\right)\right] \\
& \times P\left(\tau^{*}>T\right), \\
& H_{3} \triangleq E\left[e^{-\int_{0}^{T} \beta(t) d t} \times\left(B_{F}+c(T)\right)\right. \\
& \left.\times 1_{\left\{\tau^{*}>T, \exp \left\{-\int_{0}^{T} \beta(t) d t\right\} S(T) \leq \exp \left\{-\int_{0}^{T} r(t) d t\right\} K_{1}\right\}}\right] \\
& =\left[\left(B_{F}+c(T)\right) \cdot \exp \left\{\frac{1}{2} \sigma_{X}^{2}-G(0, T)\right\} N\left(-d_{4}\right)\right] \\
& \times P\left(\tau^{*}>T\right),
\end{aligned}
$$

where $d_{3}$ and $d_{4}$ are given by (29).
Then, the general expression of convertible bond price satisfies (28). So with (28), we can obtain the convertible bond price using the Monte Carlo simulation.

\section{Numerical Examples}

The general European options mainly take the classic B$S$ formula results as the reference prices. However, there are usually some differences between the actual transaction prices and the reference prices of the options, and it is difficult to obtain the data of the actual option prices. Therefore, we first consider some specific numerical examples under given parameters to compare the results of the actuarial pricing formula with the B-S formula and to reveal the impact of the stochastic interest rates on pricing results when the stock prices follow $\mathrm{O}-\mathrm{U}$ process. Here, it should be pointed out that if there is no arbitrage in the market, there must be at least one risk-neutral martingale measure, and the stock price process can be transformed into a martingale. In this case, the $\mathrm{O}-\mathrm{U}$ model is free of arbitrage, just as the method of B-S model is.

Now, we make numerical examples to the European options. The parameters in the financial market models are selected as follows:

$$
\begin{aligned}
& K=60, \quad T=1, \quad a=0.034, \\
& b=0.016, \quad \sigma_{s}=0.395, \quad \sigma_{r}=0.023, \\
& \alpha=0.2, \quad r=0.0245, \quad \rho=-0.58,
\end{aligned}
$$

and we obtain the option pricing results by Matlab.

As the underlying stock prices follow $\mathrm{O}$-U process and the short-term interest rates are stochastic variable, we compare the European option values generated by actuarial approach with the values generated by the classic B-S model to the European call option and the put option, as Figure 1 shows. Figure 1 shows that there are more differences between the pricing results derived by the two different methods, actuarial approach and B-S model, for the out-the-money options, while the pricing differences between the two methods are smaller for in-the-money options; in particular, the results of deep-in-the-money options are almost the same. The differences are caused by the different methods used, the underlying stock prices of $\mathrm{O}-\mathrm{U}$ process, and the stochastic short-term interest rates. Moreover, the using of different pricing models should have a larger impact which appears as negative on out-the-money options, as the exercise probabilities to out-the-money options are low, especially to deepout-the-money options, and their values mainly are the time value. However, the changes of the stock prices have more impact on the prices of deep-in-the-money options, so the pricing differences between the two different methods are much smaller.

Then, we consider the impact of stochastic interest rates on the option pricing. To this end, we compare the pricing results of the European call option derived by the actuarial approach under the constant interest rates model with the results under the stochastic interest rates model. Table 1 shows that the pricing results of the call option tend to increase with the underlying stock prices rise for the two 


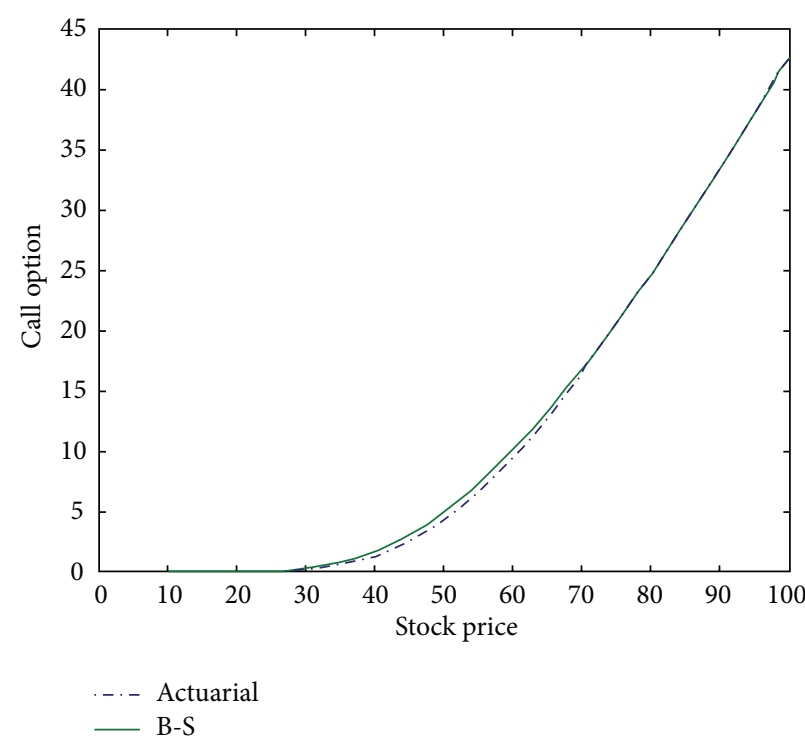

(a)

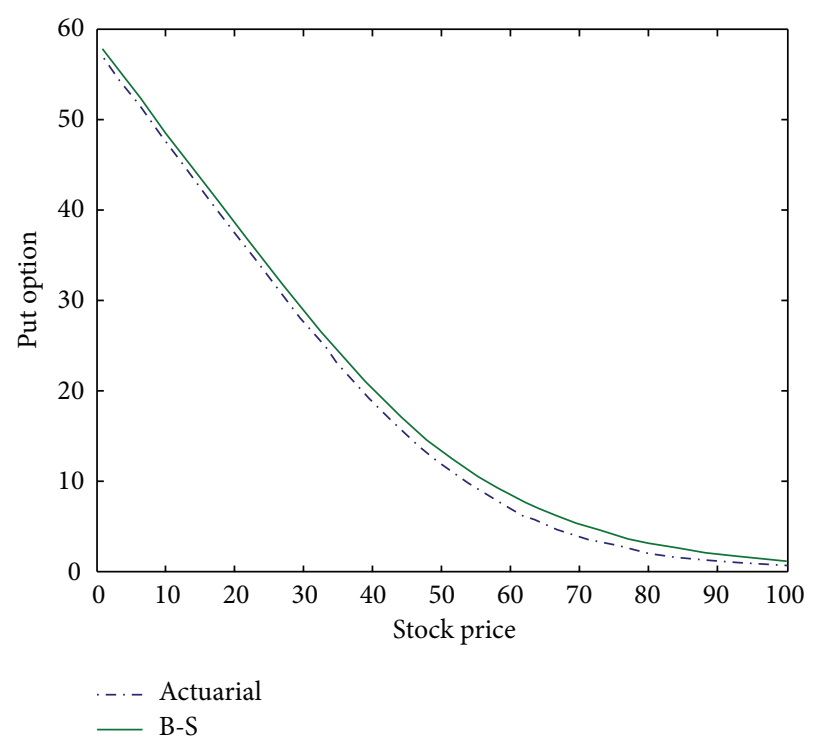

(b)

FIGURE 1: Comparison of the options values from actuarial approach under stochastic interest rates and O-U process with those from B-S formula.

TABLE 1: Comparison of the results given by the actuarial approach with stochastic interest rates and constant interest rates.

\begin{tabular}{lccccccc}
\hline Model & 40 & 45 & 50 & 55 & 60 & 65 & 70 \\
\hline $\begin{array}{l}\text { Stochastic } \\
\text { interest }\end{array}$ & 1.293 & 2.522 & 4.296 & 6.620 & 9.457 & 12.748 & 16.420 \\
$\begin{array}{l}\text { Constant } \\
\text { interest }\end{array}$ & 1.269 & 2.459 & 4.176 & 6.426 & 9.180 & 12.383 & 15.969 \\
\hline
\end{tabular}

different models. However, if the underlying stock price is fixed, the pricing result of the call under constant stochastic interest rates is lower than the pricing result under stochastic interest rates. And with the stock price increasing, the difference between the two models increases at first and then decreases. The stochastic interest rates increase the uncertainty and may increase the prices of the call option.

\section{Empirical Study}

5.1. Data and Parameters. We analyze the differences of the two models, B-S model and actuarial model, using the actual data in the Chinese stock market. However, there are only three warrants that are similar to the call option. The only difference between the warrant and the option is that the option is a standardized contract but the warrant is not. However, it would not affect the results. So we choose the data of Changhong CWB1 which is equivalent to a European call option, as its exercise ratio is $1: 1$ and the underlying stock has not paid dividend during the chosen period; then we need not adjust the prices of the stock and the warrant. The data covers the period from August 19, 2009, when the Changhong CWB1 was listed to May 20, 2010, and the time interval between each observation is one day. This means that we end up with 181 observations, and this is the longest sample available to us. The data are taken from the Shanghai Stock Exchange and are collected through the Dazhihui quote software.

Next, we need to estimate the parameters in the models. Some parameters we can get from public information are as follows: the strike price of the Changhong CWB1 is $K=5.23$, the exercise date is $T=2$ years, the interval is $\Delta t=1 / 250$, and the interest rate at 0 time is $r=0.0225$ which is the official interest rate for one-year deposits during the period 2009-2010. As for the parameters in the interest rate model, we use the daily data of 7-day repo rates in the Shanghai Stock Exchange from August 19, 2009, to May 20, 2010, and the MLE method to estimate the parameters by Matlab. The result shows that the estimated parameters of the shortterm interest rates driven by the Hull-White model in China market are $a=0.0322, b=0.1937$, and $\sigma_{r}=0.0863$. In order to estimate the parameters of the underlying stock, we use the historical data of Changhong shares during the same time to compute the results: $\sigma_{S}=0.6968$ and $\alpha=0.1359$ by the MLE method. A large number of works in the literature have revealed that the correlation of the stock price and the interest rate is weak negative [19], and we indeed get the parameter $\rho=-0.284$ during the chosen time.

5.2. Results. Having specified the data and the parameters used in the simulation procedure, we now turn to the empirical results obtained from Matlab. Figure 2 shows the comparison results among the real values, actuarial model prices, and B-S model prices. The main features are that the actuarial prices are lower than the B-S prices mostly and the actual price points are much closer to the points computed by the actuarial model than the B-S model. Moreover, we analyze the goodness of fitting by the correlation coefficients. The results show that the correlation coefficient of the actual 


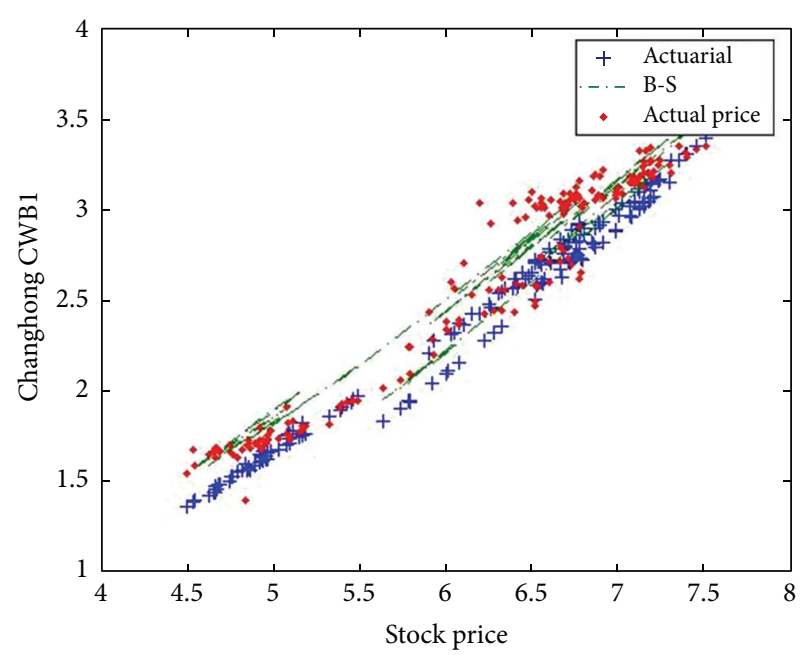

FIGURE 2: Comparison of the values among the actual data, actuarial model, and B-S model.

prices and the actuarial model prices is 0.9652, and the correlation coefficiency of the actual prices and the B-S model prices is 0.9607 which is lower than the former. It is an explicit evidence that the efficient of the actuarial model is superior to the B-S model. The analysis also shows that the correlation of the actuarial prices and the B-S prices is strong and positive as their correlation coefficient is 0.9996.

\section{Conclusions}

In the martingale pricing method, the prices of the financial derivative securities (or contingent claims) are obtained by discounting their expectations of future cash flows which are computed with risk neutrality. It is valid for the complete market, because the unique equivalent martingale exists. However, if the market is not arbitrage-free and incomplete, the equivalent martingale measure does not exist or exists but is not unique; then it is hard to price by the martingale pricing method. As no economic assumptions are involved, the actuarial approach is valid for incomplete markets as well as for complete markets and needs not find an equivalent martingale measure for pricing. This paper discusses the pricing problem of the European options and convertible bonds using the actuarial approach. We get the pricing formula of the European options, extend the pricing results to the case with continuous dividend, and then derive the call-put parity relation. Furthermore, we get the general expression of convertible bond price. Finally, we conduct a comparative analysis of numerical simulation and make an empirical analysis between the B-S model and the actuarial model using the actual data in the Chinese stock market. The empirical results show that the efficiency of actuarial model is superior to the B-S model.

\section{Acknowledgments}

The authors would like to express gratitude for the support given by the National Natural Science Foundation of China (no. 71201013), the National Natural Science Innovation Research Group of China (no. 71221001), the National Science Fund for Distinguished Young Scholars of China (no. 70825006), and the Humanities and Social Sciences Project of the Ministry of Education of China (no. 12YJC630118).

\section{References}

[1] F. Black and M. Scholes, "The pricing of options and corporate liabilities," Journal of Political Economy, vol. 81, no. 3, pp. 637654, 1973.

[2] R. C. Merton, "Theory of rational option pricing," Bell Journal of Economics and Management Science, vol. 4, no. 1, pp. 141-183, 1973.

[3] J. C. Cox, S. A. Ross, and M. Rubinstein, "Option pricing: a simplified approach," Journal of Financial Economics, vol. 7, no. 3, pp. 229-263, 1979.

[4] D. Duffie, Security Markets: Stochastic Models, Academic Press, San Diego, Calif, USA, 1998.

[5] R. H. Liu and J. L. Zhao, "A lattice method for option pricing with two underlying assets in the regime-switching model," Journal of Computational and Applied Mathematics, vol. 250, pp. 96-106, 2013.

[6] M. Ammann, A. Kind, and C. Wilde, "Simulation-based pricing of convertible bonds," Journal of Empirical Finance, vol. 15, no. 2, pp. 310-331, 2008.

[7] R. X. Xu, "A lattice approach for pricing convertible bond asset swaps with market risk and counterparty risk," Economic Modelling, vol. 28, no. 5, pp. 2143-2153, 2011.

[8] H. Follmer and D. Sondermann, Contributions to Mathematical Economics, North Holland, New York, NY, USA, 1986.

[9] M. Bladt and T. H. Rydberg, "An actuarial approach to option pricing under the physical measure and without market assumptions," Insurance, vol. 22, no. 1, pp. 65-73, 1998.

[10] H. F. Yan and S. Y. Liu, "New method to option pricing for the general Black-Scholes model: an actuarial approach," Applied Mathematics and Mechanics, vol. 24, no. 7, pp. 730-738, 2003.

[11] W. Zhao and T. M. He, "Model of option pricing driven by fractional Ornstein-Uhlenback process," Chinese Journal of Management Science, vol. 15, pp. 1-5, 2007.

[12] L. Dong, "An empirical test of mean-reversion hypothesis of short-term interest rate in China," The Journal of Quantitative and Technical Economics, vol. 11, pp. 151-160, 2006.

[13] C. Christiansen, "Mean reversion in US and international short rates," North American Journal of Economics and Finance, vol. 21, no. 3, pp. 286-296, 2010.

[14] J. Liu, G. H. Deng, and X. Q. Yang, "Pricing reload options with stochastic interest rate under Ornstein-Uhlenbeck processes," Journal of Engineering Mathematics, vol. 24, no. 2, pp. 237-241, 2007.

[15] S. N. Chen, Financial Engineering, Fudan University Press, Shanghai, China, 2002.

[16] M. J. Brennan and E. S. Schwartz, "Convertible bonds: valuation and optimal strategies for call and conversion," The Journal of Finance, vol. 32, pp. 1699-1715, 1977.

[17] J. E. Ingersoll Jr., "A contingent-claims valuation of convertible securities," Journal of Financial Economics, vol. 4, no. 3, pp. 289321, 1977. 
[18] F. H. Wen, Z. Li, C. H. Xie, and D. Shaw, "Study on the fractal and chaotic features of the Shanghai composite index," FractalsComplex Geometry Patterns and Scaling in Nature and Society, vol. 20, pp. 133-140, 2012.

[19] F. H. Wen and Z. F. Dai, "Modified Yabe-Takano nonlinear conjugate gradient method," Pacific Journal of Optimization, vol. 8, no. 2, pp. 347-360, 2012. 


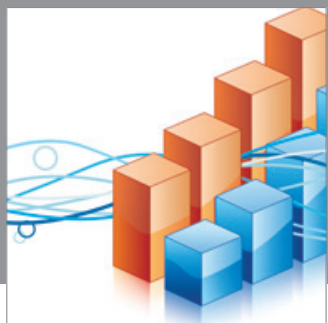

Advances in

Operations Research

mansans

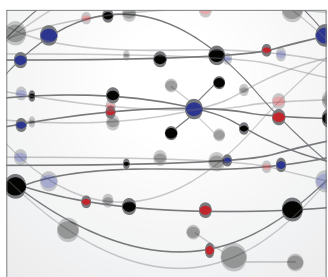

The Scientific World Journal
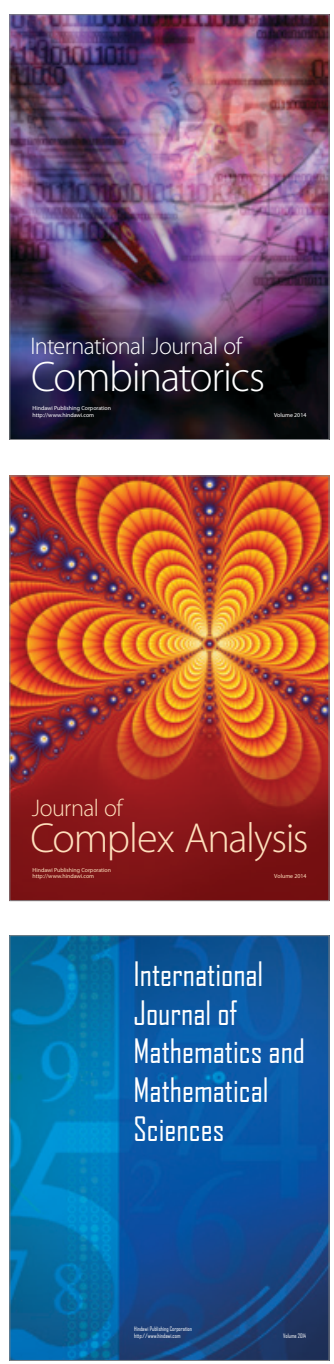
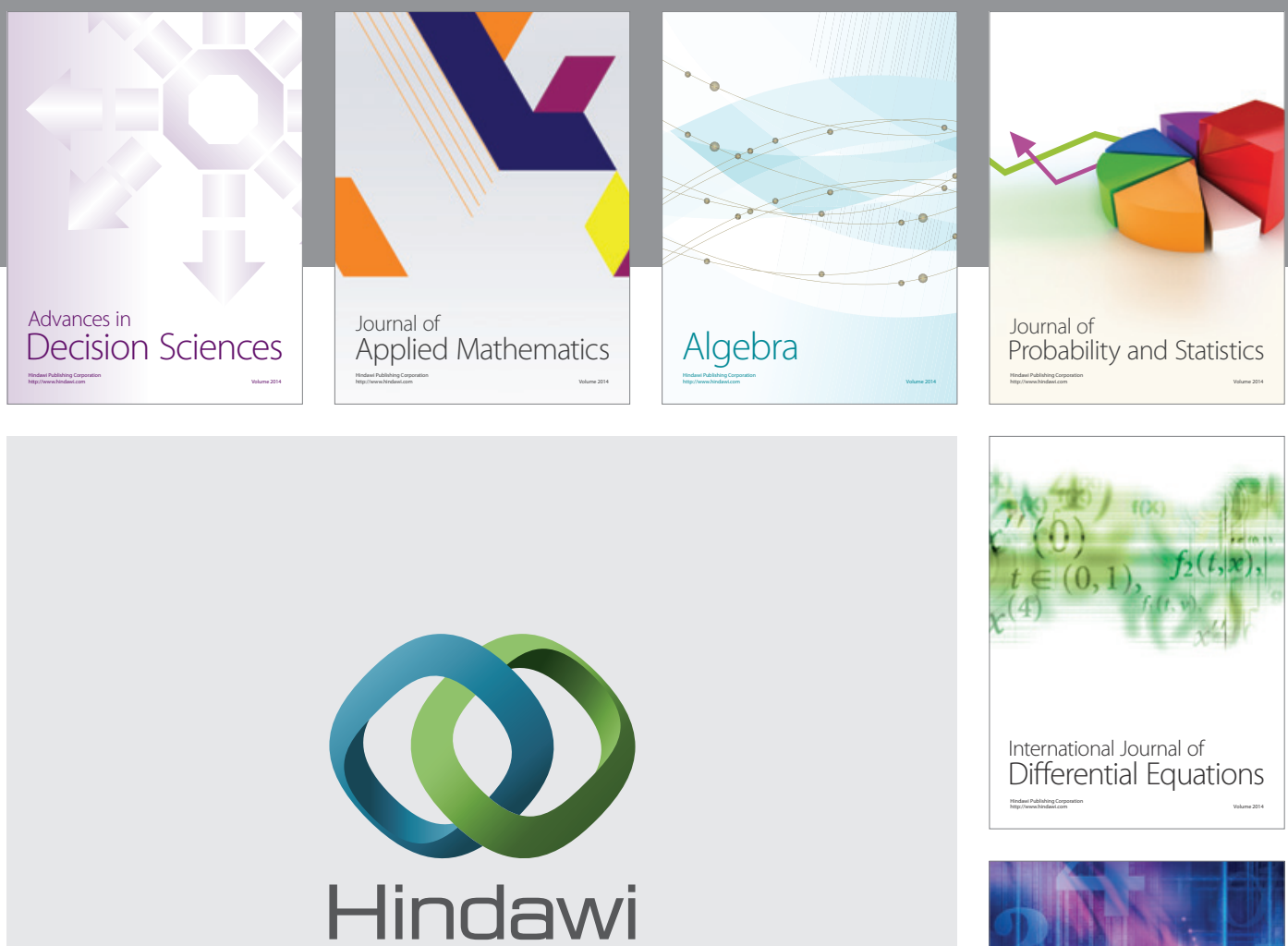

Submit your manuscripts at http://www.hindawi.com
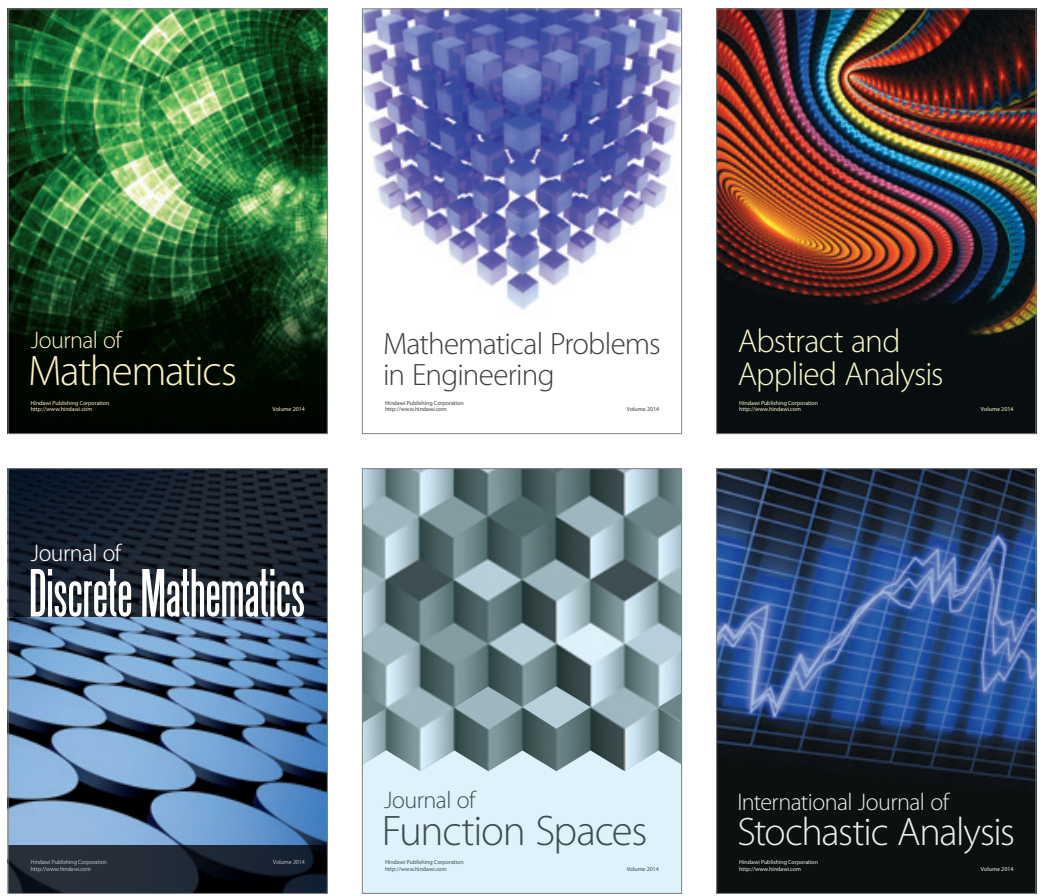

Journal of

Function Spaces

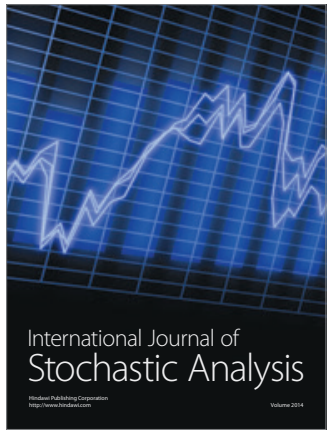

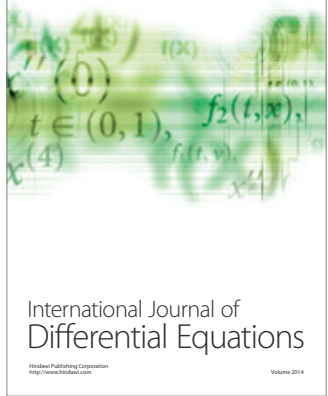
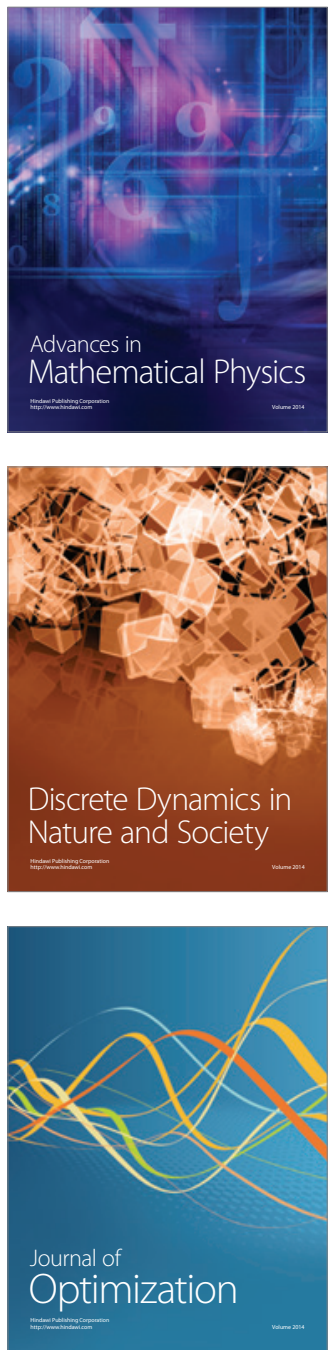